\title{
Anabases
}

ANABASES Traditions et réceptions de l'Antiquité

1 | 2005

Varia

\section{Antiquité et actualité grecques, Révolution française et nation allemande dans le roman Hypérion de Hölderlin (1797-1799)}

\section{Lucien Calvié}

\section{OpenEdition}

Journals

Édition électronique

URL : http://journals.openedition.org/anabases/1274

DOI : 10.4000 /anabases. 1274

ISSN : 2256-9421

Éditeur

E.R.A.S.M.E.

\section{Édition imprimée}

Date de publication : 1 mars 2005

Pagination : 45-57

ISSN : $1774-4296$

\section{Référence électronique}

Lucien Calvié, «Antiquité et actualité grecques, Révolution française et nation allemande dans le roman Hypérion de Hölderlin (1797-1799) », Anabases [En ligne], 1 | 2005, mis en ligne le 01 septembre 2011, consulté le 20 octobre 2019. URL : http://journals.openedition.org/anabases/1274 ; DOI 10.4000/anabases.1274 


\section{Antiquité et actualité grecques, Révolution française et nation allemande dans le roman Hypérion de Hölderlin (1797-1799)}

1.

D ÈS LA PREMIÈRE page de ce roman par lettres à la mode du XVIII e siècle, l'essentiel est annoncé. Hypérion, un jeune Grec moderne des Cyclades, écrit à son ami Bellarmin, un Allemand en quelque sorte générique, qui restera silencieux pendant tout le roman et n'aura aucun rôle dans l'action. Tiré d'une belle rêverie antique par le cri funèbre du chacal au milieu des ruines de Corinthe, Hypérion dit à Bellarmin la situation misérable de sa patrie, la Grèce moderne soumise à l'humiliation du joug ottoman:

«Heureux celui dont le cœur tire joie et force de la prospérité de sa patrie! Pour moi, quand on me parle de la mienne, c'est comme si on m'avait jeté dans un bourbier, refermé sur la tête le couvercle d'un cercueil; et de m'entendre appeler «Grec», je me crois le chien auquel on passe son collier autour du cou.» (136) ${ }^{1}$.

Mais le sort malheureux du héros et de sa patrie paraît s'étendre - au prix d'un décalage chronologique entre 1770, année de la première révolte grecque, décrite dans le roman, contre la domination ottomane, et la dernière décennie du XVIIIe siècle - à la patrie de son lointain correspondant, plus précisément à ces philosophes idéalistes qui sont pourtant la gloire et l'illustration de l'Allemagne. Hypérion ajoute en effet:

«Or, sache-le, mon Bellarmin: chaque fois que de tels propos m’ont échappé, chaque fois que j'ai pleuré de dépit, j'ai vu venir à moi les sentencieux personnages qui hantent votre Allemagne, ces malheureux qui n'aiment tant les âmes souffrantes que pour leur appliquer leurs maximes: et de s'en donner à cœur joie, et de me dire, entre autres bons conseils: "Plutôt que de gémir, si tu agissais?"” (136-137).

Les indications de page entre parenthèses se réferent aux Euvres de Hölderlin: HÖLDERLIN, Euvres dir. P. JACCOTTET, Paris, Gallimard, 1967. 
Bien plus qu’à Kant, déjà âgé de soixante-cinq ans en 1789 et dont l'éloge de la «bonne volonté», définie comme seule "absolument bonne», ne débouche pas nécessairement sur un appel à l'action et ne fait même pas du but de l'action le critère de la valeur morale de celle-ci, plus aussi qu'à Hegel et à Schelling, les condisciples et amis de Hölderlin au séminaire protestant (Stift) de Tubingue, en Wurtemberg, de 1788 à 1793, cette critique de l'apologie de l'action fait penser à Fichte dont Hölderlin, en 1794-1795, a suivi avec passion l'enseignement à l'université d'Iéna ${ }^{2}$.

Fichte, né en 1762 et de huit ans seulement l'âné de Hölderlin, avait, dans sa Doctrine de la science (Wissenschaftslehre) publiée en 1794 et 1795, insisté sur la liberté inconditionnée du Moi et fait l'éloge de la volonté humaine agissante triomphant des obstacles dressés par la Nature et le monde. Ajoutées à deux textes directement politiques de 1793 et 1794 sur la liberté de pensée et d'expression (Revendication pour la liberté de penser) et sur la Révolution française (Contributions destinées à rectifier le jugement du public sur la Révolution française), défendue par Fichte contre les tenants allemands d'un conservatisme historiciste inspiré de Burke, ces publications avaient valu au philosophe une durable réputation de «jacobinisme».

La première lettre du roman s'achève sur un regret: si seulement, assure Hypérion à son ami, j'avais pu avoir la sagesse de ne pas agir! Et il souhaite un retour enfin apaisé à «l'immuable et silencieuse beauté de la Nature» (137). Cette aspiration au repos dans la beauté de la Nature représente l'antithèse de la tension engendrée par l'intellectualisme, le subjectivisme et le volontarisme fichtéens. C'est d'ailleurs Hölderlin qui a appelé son ami Schelling, dès 1794-1795, à rompre avec la pensée de Fichte et à lui opposer la philosophie de la Nature. Hypérion peut ainsi se lire comme une confrontation critique avec la première pensée de Fichte ${ }^{3}$.

Cette mise en cause n'implique cependant pas une critique du «jacobinisme», c'est-à-dire du soutien à la Révolution française dans ce qu'elle a d'essentiel. Au contraire, le «jacobinisme» de Hölderlin - pour reprendre une formule un peu provocatrice utilisée voici un quart de siècle par le germaniste Pierre Bertaux ${ }^{4}$, professeur à la Faculté des Lettres de Toulouse juste avant la deuxième guerre mondiale, résistant de la première heure à l'Occupation et Commissaire de la République dans la même ville de 1944 à 1946 - est sans doute plus constant que celui de Fichte mais son fondement conceptuel est assez différent: plus proche des sources antiques, plus moniste ou spinoziste aussi - Hölderlin a lu Spinoza dès le

2 Voir S. WACKWITZ, Friedrich Hölderlin, Stuttgart, Metzler, 1985, p. 21 et 72-77.

3 Voir J. RIVELAYGUE, Leçons de métaphysique allemande, t. I, De Leibniz à Hegel, Paris, Librairie Générale Française (Le Livre de Poche), 2003 (1 édition, Paris, Grasset, 1990), en particulier p. 199-228: «La rencontre avec Hölderlin».

4 Voir P. BERTAUX, Hölderlin und die Französische Revolution, Francfort-sur-le-Main, Suhrkamp, 1969. 
séminaire de Tubingue -, et plus soucieux de retour à l'unité fondamentale de l'Être que de différenciation dynamique entre la pensée et le monde, ou le réel.

Dès la première lettre du roman se trouvent annoncées la formation et l'évolution d'Hypérion telle qu'elles apparaissent par la suite. Dialectiquement, trois phases de contemplation méditative précèdent deux phases d'action politique ou leur succèdent. Voici, brièvement résumées, ces cinq phases successives. La première phase contemplative, c'est celle de la jeunesse d'Hypérion et de son heureuse éducation par un sage de style et de mœurs antiques, Adamas. La première phase active, c'est la rencontre d'Hypérion avec son aîné Alabanda; celui-ci, partisan d'un retour aux vertus antiques, a fondé une ligue patriotique de lutte contre la domination ottomane, la ligue de la Némésis, aux idées quelque peu fichtéennes et aux allures plutôt «jacobines». La deuxième phase contemplative, après la rupture avec Alabanda, est marquée par un retour d'Hypérion à la Nature et par sa découverte de l'amour auprès de la belle et sage Diotima, autour de l'idée d'une renaissance de la beauté et de l'équilibre de l'ancienne Athènes. La deuxième phase active décrit, après la réconciliation avec Alabanda, la participation d'Hypérion à la première guerre d'indépendance des Grecs contre l'Empire ottoman en 1770, à l'occasion d'une guerre russo-turque (1768-1774), jusqu'au siège, à la prise et au pillage de Mystra, près de l'antique Sparte. La troisième phase méditative, enfin, après les déceptions et l'échec de cette guerre, évoque un nouveau retour à la contemplation de la Nature.

Au-delà de la confrontation critique immédiate avec la pensée de Fichte, cette succession dialectique de phases contemplatives et actives peut faire l'objet d'une interprétation plus large, en liaison avec la situation personnelle et intellectuelle de Hölderlin dans les intenses débats de son temps autour de l'idéalisme philosophique allemand de Kant à Hegel, en passant par Schiller, Fichte et Schelling. Hölderlin, en effet, a lu Kant; il écoute avec passion les cours de Fichte à Iéna; il trouve en Schiller un aîné qu'il admire et qui, parfois, le soutient dans ses débuts littéraires; quant à Hegel et à Schelling, il entretient avec eux, depuis leur scolarité commune à Tubingue, une relation intense faite de longs échanges épistolaires, à propos des cours de Fichte en particulier, et de lectures réciproques commentées.

D'un côté, en effet, l'amour pour Diotima, plusieurs fois explicitement identifiée à la nature, représente une sorte de pôle panthéiste ou spinoziste, un refus de la subjectivité et un abandon à la nature et au monde dans leur totalité, par-delà la scission introduite par la conscience, le Moi pensant. À l'inverse, l'amitié pour Alabanda et le combat politique et militaire un temps mené en sa compagnie figurent l'autre pôle possible de la pensée, celui de Fichte et de l'affirmation dynamique du Moi pensant et agissant face au réel. Ces deux tentations conduisent, dans le roman, à un double échec: Diotima, en effet, devient inaccessible et s'abandonne à la mort au moment même où Hypérion, déçu par la violence d'Alabanda et de ses partisans lors de la prise et du pillage de Mystra, revient vers elle. 
Il est remarquable que le dépassement de la contradiction entre la position panthéiste ou spinoziste (Diotima) et la position subjectiviste ou fichtéenne (Alabanda) se trouve, chez Hölderlin, dans une référence à la pensée antique, précisément à Platon et à sa théorie du Beau et de la poésie qui tente de le représenter. Au cours d'une des étapes de la rédaction du roman, en effet, à la fin de l'année 1795, Hölderlin avait écrit un projet de préface dans lequel il notait ceci:

«Les contradictions, les égarements [...] de cet Hypérion agaceront peut-être [...] L'unité de l'âme, l'Être [...] est perdu pour nous [...]Nous nous arrachons au paisible én kai pan du monde pour le rétablir par nous-mêmes. Nous avons rompu avec la nature, et ce qui était naguère [...] un, maintenant s'est fait contradiction [...] Souvent il nous semble que le monde est tout et que nous ne sommes rien, mais souvent aussi que nous sommes tout, et le monde rien. Hypérion a connu aussi cet écartèlement entre les deux extrêmes.

Mettre fin à ce combat entre nous et le monde, [...] nous unir avec la Nature en un Tout infini, tel est le but de toutes nos aspirations [...].

Nous n'aurions aucune idée de cette paix infinie [...], nous n'aspirerions nullement à nous unir avec la Nature, nous ne penserions ni n'agirions [...], si cette union infinie, si cet Être au seul sens du mot, n'existait pas. Il existe - comme Beauté; pour parler avec Hypérion, un nouveau royaume nous attend, où la Beauté sera reine.

Je crois qu'à la fin nous nous écrierons tous: saint Platon, pardonne-nous! nous avons gravement péché contre toi.» (1150).

L'existence platonicienne du Beau et de la poésie qui en constitue l'approche progressive et approximative apparaît donc, sous la plume de Hölderlin travaillant à Hypérion, comme le dépassement possible de la contradiction entre la position spinoziste (le monde comme «tout» et le Moi pensant comme «rien») et la position fichtéenne (le monde comme «rien» et le Moi pensant comme «tout»), contradiction figurée, dans le roman, par le vain et épuisant affrontement entre Diotima et Alabanda.

Plus généralement encore, c'est autour d'une référence précise à la pensée de la Grèce antique, celle d'Héraclite en l'occurrence, cité par Platon, que Hölderlin parâtt rechercher un possible dépassement de la contradiction entre un idéalisme subjectiviste et actif de type fichtéen, mettant l'accent sur la séparation et la différence entre le Moi et le monde, et une position contemplative de type spinoziste, privilégiant l'unité primordiale de l'un et de l'autre.

C'est là du moins ce qui ressort d'une lettre particulièrement importante d'Hypérion, dont il sera encore question par la suite. Dans une discussion avec Diotima et des amis sur «l'excellence des anciens Athéniens» (199), Hypérion affirme en effet que l'art grec ancien est «le premier enfant de la beauté humaine» et que la religion en est le «second» (201); quant à la philosophie comme conscience réflexive, elle n'arrive selon lui à l'existence que par la poésie qui est à la fois "le commencement et la fin de cette science [la philosophie]» (202). Et Hypérion de conclure sa démonstration sur l'unité fondamentale du Beau dans l'art, la religion, la 
poésie et la philosophie des Grecs anciens par une citation d'Héraclite, d'ailleurs mentionnée sous une forme différente par Platon dans Le Banquet ${ }^{5}$ :

«[...] Seul un Grec pouvait inventer la grande parole d'Héraclite, én diaféron éauto l'Un distinct en soi-même -, car elle dit l'essence de la beauté, et avant qu'elle fût inventée, il n'y avait pas de philosophie.» (203).

Il ne s'agit donc pas, pour Hölderlin-Hypérion, de faire un choix réducteur entre contemplation moniste ou panthéiste (Diotima) et idéalisme dualiste et activiste (Alabanda), mais de concevoir et, éventuellement, de vivre l'unité profonde de ces deux attitudes humaines en apparence opposées, unité représentée par la formule souvent - et très diversement - citée d'Héraclite sur l'unité dialectique de la différence et de l'identité.

\section{2.}

La troisième et dernière phase contemplative de l'évolution d'Hypérion ne représente pas vraiment une clôture absolue dans la mesure où la dernière lettre du roman s'achève sur une formule ouverte: «j'en dirai plus une autre fois» ("Nächstens mehr», 273) qui laisse supposer un possible développement ultérieur, l'histoire personnelle d'Hypérion n'ayant pas davantage de fin, dans le récit, que l'Histoire en général.

Cette ouverture sur l'avenir trouve une possible illustration dans le fait historique suivant, à la fois tout à fait imprévisible, pour Hölderlin, à la fin du XVIIIe siècle, et sans doute secrètement espéré par lui: au début des années 1820, au moment de la deuxième et décisive guerre d'indépendance grecque contre l'Empire ottoman, des philhellènes allemands appuyèrent leur propagande humanitaire et politique sur des extraits d'Hypérion dont une réédition avait paru en 1822. Parmi ces philhellènes, on peut citer Wilhelm Waiblinger, auteur de la première biographie de Hölderlin ainsi que d'une mise en ouvre de sa vie et des sa personnalité dans un roman intitulé Phaéton, en 1822 également. On peut également mentionner Rudolf Lohbauer qui par la suite, au début des années 1830, devait jouer un rôle très actif dans le développement d'une opposition à tendance parfois républicaine, en Wurtemberg, autour du journal radical La Sentinelle (Der Hochwächter) ${ }^{6}$.

5 «C'est peut-être ce qu'Héraclite voulait dire, bien qu'il ne se soit pas bien expliqué, quand il affirmait que l'unité s'opposant à elle-même produit l'accord, comme l'harmonie de l'arc et de la lyre." (Platon, Banquet, trad. et notes par É CHAMBRY, Paris, Garnier-Flammarion, 1964, p. 46).

6 Voir A. KELLETAT, "Rudolf Lohbauers Hyperion-Brief und Hyperion-Bild aus dem Jahre 1824”, Hölderlin-Jahrbuch 10 (1957), p. 182-190 et L. CALVIÉ, Les Intellectuels allemands, les réalités politiques allemandes et l'idée de révolution (1789-1844) - De la Révolution française aux débuts du marxisme, thèse de doctorat d'État, Paris III, 1979, p.433-435. 
Et c'est bien de l'Histoire en général et de certains développements historiques particuliers qu'il est question dans le roman de Hölderlin. Mais l'Histoire, ici, ce n'est pas seulement le passé, éloigné dans l'Antiquité ou beaucoup plus proche, c'est aussi l'Histoire en train de se faire, l'actualité immédiate de cette dernière et très mouvementée décennie du XVIII siècle qui, pour nous, aujourd'hui, est de l'Histoire, vivante encore certes, mais, pour Hölderlin comme pour son ami Hegel ou pour Fichte, était une actualité aussi brûlante que bouleversante.

La structure du roman repose en effet sur la juxtaposition à peu près permanente de trois «moments» ou de trois "temps» historiques. Tout d'abord le «moment» fortement idéalisé de la Grèce antique, organisé autour de l'image d'Athènes au temps de sa splendeur. Ensuite le «moment» du texte lui-même, celui de l'action du roman par lettres et de la première lutte de la Grèce moderne contre la domination ottomane en 1770, l'année même de la naissance de Hölderlin. Et enfin le «moment» de l'actualité immédiate, celui de la rédaction et de la publication d'Hypérion, c'est-à-dire le «moment» de la Révolution française et de ses conséquences en Europe et en Allemagne.

La première révolte grecque contre les Turcs - sur laquelle Hölderlin s'est documenté, peut-être grâce à une traduction allemande du tome premier, paru en 1782, du Voyage pittoresque de la Grèce du comte de Choiseul-Gouffier, mais la recherche n'est guère bavarde à ce sujet ${ }^{7}$ - est antérieure d'un quart de siècle environ à la période de rédaction du roman, d'un premier manuscrit en 1792 aux deux volumes définitifs parus en 1797 et 1799, en passant par divers fragments en vers et en prose rédigés, et parfois publiés, entre 1794 et 1796 . Ce "temps» de la rédaction, qui est aussi celui de la Révolution française, et celui, fictif, du roman, c'est-à-dire de la révolte de 1770, sont confrontés, explicitement ou implicitement, à travers de longs développements théoriques ou à travers le mode d'expression de Hölderlin, tout imprégné de références antiquisantes, au «temps» le plus reculé, celui de la Grèce antique et particulièrement de la polis démocratique et harmonieuse dont Athènes fournit le modèle à la fois réel et idéal.

Mais, à travers la référence romanesque à la révolte de 1770 comme aussi à travers la référence à la polis athénienne, c'est toujours de l'actualité immédiate qu'il est question, c'est-à-dire de la Révolution française et de ses conséquences pour l'Allemagne comme nation. La question qui préoccupe Hypérion, et Hölderlin, est celle-ci: comment faire revivre dans le monde moderne l'état d'harmonie et d'équilibre, mais aussi de liberté individuelle et politique qui caractérisait, du moins Hölderlin le suppose-t-il idéalement, les Athéniens de l'époque classique? Et encore: comment mettre en œuvre de façon consciente et rationnelle ce que les Athéniens de

7 Voir M. MONTGOMERY, F. Hölderlin and the German Neo-Hellenic Movement, 2 parts, Oxford, Humphrey Milford-Oxford University Press, 1923. 
l'époque classique avaient atteint, idéalement, de façon spontanée et naturelle: le bonheur dans l'équilibre, la beauté et la liberté?

Ce sont ces questions qui sont abordées par Hypérion, Diotima et leurs amis au centre à peu près exact du roman, à la fin du premier volume, dans la longue discussion de type platonicien - Hölderlin a beaucoup et très tôt lu Platon - déjà évoquée sur «l'excellence des anciens Athéniens» comparée aux défauts des autres peuples. Cette double discussion, d'une part, sur le lien entre la beauté des anciens Grecs et de leurs œuvres d'art et la liberté individuelle et politique dont ils jouissaient et, d'autre part, sur leur supériorité par rapport aux autres peuples anciens et modernes, n'était pas vraiment nouvelle dans la vie intellectuelle allemande de la deuxième moitié du XVIII e siècle ${ }^{8}$. Elle avait en particulier été abordée par Winckelmann, de façon fugitive, dans ses Réflexions sur l'imitation des auvres grecques de 1755 et, de façon plus développée, en 1764, dans son Histoire de l'art de l'Antiquité.

Du reste, Hölderlin, dans Hypérion, cite presque textuellement Winckelmann. À ses amis qui, reprenant fidèlement l'argumentation de Winckelmann en 1764 ', estiment que «l'excellence» des anciens Athéniens pouvait être due au climat, ou à l'art, ou à la philosophie, ou encore à la religion, ou enfin à l'organisation politique, Hypérion-Hölderlin répond qu'ils prennent "les effets pour la cause» (199). Selon lui, tous ces éléments apparemment explicatifs s'expliquaient eux-mêmes, en fait, par une liberté innée, spontanée, naturelle et fondamentale que l'éducation se contentait d'entretenir et de développer. La liberté spontanée de l'Athénien s'opposait ainsi à l'attitude naturelle de «l'Égyptien», qui "supporte sans souffrance le despotisme et l'arbitraire», et à celle, tout aussi innée, du "Fils du Nord», qui admet sans broncher «le despotisme de la loi, l'injustice légale» (202).

Il faut s'interroger sur les sources antiques de cette discussion, que Hölderlin a pu connaître directement ou par le biais de textes modernes les reprenant. Platon a fait de nombreuses remarques sur la psychologie des différents peuples - les Perses et les Grecs surtout -, ses conséquences politiques et ses liens avec le climat. De même, on trouve chez Hippocrate l'ébauche d'une "théorie des milieux et des climats» à la Montesquieu. Tout cela se retrouve, systématisé, au livre VII de la Politique d'Aristote. Celui-ci reprend l'argumentation de Platon sur le climat de la Grèce comme élément favorable à l'équilibre et au courage, car «intermédiaire» entre les chaleurs torrides et les grands froids. Aristote, en effet, voit chez les Grecs, en "position géographiquement intermédiaire», la synthèse heureuse entre l'intelligence et la technique,

8 Voir L. CALVIÉ, “Antiquité et Révolution française dans la pensée et les lettres allemandes à la fin du XVIII e siècle”, Annales Historiques de la Révolution française 317 (1999/3), p. 455-475.

9 Winckelmanns Werke in einem Band, hrsg. von Helmut HOLTZHAUER, Berlin et Weimar, Aufbau, 1982, p. 179. 
d'une part, et, d'autre part, le courage physique. Cette synthèse est, selon lui, au fondement de leur liberté individuelle et politique. Elle les met au-dessus, à la fois, des "peuples des régions froides et de l'Europe», courageux mais peu intelligents et peu habiles et dont la liberté ne peut donc être que «relative», et plus encore au-dessus des "peuples de l'Asie», intelligents et habiles, certes, mais dénués de courage et donc portés à «la soumission et à l'esclavage» 10 .

La proximité, jusque dans le détail, entre la discussion hölderlinienne et ces possibles sources est évidente, en particulier au sujet de la supériorité des Grecs, du point de vue de la liberté, sur les "peuples du Nord ou de l'Europe» et sur les «Égyptiens» ou les "peuples d'Asie». Une différence fondamentale, cependant, s'impose. Hypérion, porte-parole de Hölderlin, refuse en effet d'expliquer par le milieu et le climat la liberté individuelle et politique et le degré qu'elle peut atteindre chez les différents peuples. Alors que, chez Aristote et ses prédécesseurs, le climat apparaissait comme une "cause» essentielle de la liberté, Hypérion rejette cette explication matérielle et fait remarquer à ses amis la triste différence entre la soumission des Grecs modernes au joug ottoman et, sous le même climat et dans le même milieu, la belle liberté des anciens Athéniens:

«Quant à celui qui attribue au climat cette excellence [de la liberté chez les anciens Athéniens], a-t-il réfléchi que nous vivons sous le même ciel?» (199)

En rejetant ainsi l'explication de la liberté individuelle et politique par un facteur matériel, celui du milieu et du climat, Hölderlin radicalise l'argumentation antique et la rapproche de celle, plus abstraite et plus générale, des révolutionnaires français et de leurs émules «jacobins» en Allemagne, ses contemporains, avec cependant, dans le texte d'Hypérion, quelque chose de moins volontariste et de plus déterministe, voire fataliste. Par cette double critique du déterminisme matériel de type antique et du volontarisme des révolutionnaires français, qui est aussi en partie celui de Fichte au même moment, Hölderlin est peut-être à la recherche, ici, d'un possible dépassement théorique de l'antinomie, dont on retrouve le développement à la fin du XVIII siècle et au début du suivant chez un Schiller ou un Benjamin Constant, entre la «liberté selon les Anciens», c'est-à-dire civique, politique, collective et héroïque, d'une part, et, d'autre part, la «liberté selon les Modernes», conçue comme plus civile, individuelle et jouisseuse.

Il reste qu'il existe, selon Hypérion-Hölderlin, des peuples naturellement faits pour la liberté, et donc aussi pour la beauté et le bonheur, et des peuples naturellement inaptes à cette même liberté et à ses heureuses conséquences. En affirmant cela, Hölderlin revient sur une discussion récurrente dans l'intellectualité allemande face à la Révolution française, cette question que formulaient avec acuité, en 1794, les Lettres sur l'éducation esthétique de Schiller: l'éducation esthétique,

10 Voir Aristote, Politique, t. III, $1^{\mathrm{e}}$ partie (livre VII), trad. J. AUBONNET, Paris, Les Belles Lettres, 1986, p. 75-76 et 173-176. 
morale et civique peut-elle permettre de faire l'économie d'une révolution antidespotique violente et même sanglante dont la France vient de procurer le modèle ou le contre-modèle?

La réponse de Hölderlin a le tranchant d'un couperet de guillotine: une telle éducation n'a de sens que pour un peuple naturellement libre (les «anciens Athéniens») et non pour un peuple naturellement esclave (les «Égyptiens») ou pour un peuple d'exécutants soumis au triste "despotisme de la loi» (les «Fils du Nord»). Le rapprochement s'impose avec cette formule de Rousseau que Hölderlin avait d'ailleurs choisie comme épigraphe, avec les deux phrases qui la précèdent, à son «Hymne à l'humanité» de 1791 :

«Les âmes basses ne croient point aux grands hommes: de vils esclaves sourient d'un air moqueur à ce mot de liberté»" 11 .

3.

Dès lors se pose la question, - face au modèle idéal de l'Athènes antique comme face au modèle réel et immédiat de la France "révolutionnée» - de l'aptitude des Allemands à la liberté individuelle et politique, et donc à la beauté et au bonheur, c'est-à-dire aussi la question de la nation allemande et de son destin.

En 1840, trois ans avant la mort de Hölderlin, un démocrate lui aussi d'origine wurtembergeoise, le poète Georg Herwegh, publie dans la revue radicale La Galerie populaire allemande (Die deutsche Volkshalle) dirigée par J.G.A. Wirth, un des dirigeants du libéralisme allemand dans sa nuance à la fois nationaliste et démocratique, un article dans lequel il estime que Hölderlin est devenu «fou» à cause des misérables réalités politiques de sa patrie allemande ${ }^{12}$. Herwegh, au moment de ses études au séminaire (Stift) de Tubingue en 1835-1836, avait fait la connaissance du "pauvre Hölderlin», le poète retiré dans sa tour au bord du Neckar. Herwegh cite à l'appui de son affirmation des causes politiques de la «folie» de Hölderlin un passage de l'avant-dernière lettre d'Hypérion, cette lettre qui ne concerne pas la Grèce antique, ni la Grèce moderne, mais explicitement l'Allemagne et ses habitants, et qui commence par ces formules d'une dureté extrême, égale à celle de Rousseau :

«C'est ainsi que j'arrivai en Allemagne - écrit Hypérion à Bellarmin - [...]. Des barbares de longue date, rendus plus barbares encore par leur zèle, leur science et leur religion même $[\ldots] »(267)$

11 J.-J. ROUSSEAU, Du Contrat social, précédé de "La démocratie selon Rousseau" par J.-P. SIMEON, Paris, Seuil, 1977, p. 261.

12 Voir K. KANZOG, "Bemerkungen zur Hölderlin-Rezeption im Vormärz", Wissenschaftliche Zeitschrift der Friedrich Schiller Universität Jena, Ges.- und Sprachwiss. Reihe 21 (1972), p. 471-477. 
Ce n'est pas ce début que cite Herwegh mais un autre passage, un peu plus loin, où il est question de l'effroyable isolement des poètes et des intellectuels en général en Allemagne :

«Le spectacle qu'offrent vos poètes, vos artistes, tous ceux qui estiment encore le génie, aiment et cultivent la beauté, n'est pas moins navrant. Les infortunés! ils vivent dans le monde comme des étrangers dans leur propre maison [...]» (269) ${ }^{13}$.

En mars 1843, trois mois environ avant la mort de Hölderlin le 7 juin de la même année, un hégélien de gauche, le très actif journaliste Arnold Ruge, veut démontrer à son ami Karl Marx, qui envisage déjà le possible dépassement de la révolution politique, ou bourgeoise, dans la révolution sociale, ou prolétarienne, qu'il n'y a décidément rien de bon, politiquement, à attendre de l'Allemagne et des Allemands ${ }^{14}$. Dans ce dessein, il ne trouve rien de mieux que de lui citer un passage, plus connu que le précédent, de la même avant-dernière lettre d'Hypérion sur les Allemands et l'Allemagne :

«C'est une dure parole que je vais dire, et je la dirai pourtant, parce qu'elle est véridique: on ne peut concevoir de peuple plus déchiré que les Allemands. Tu trouveras parmi eux des ouvriers, des penseurs, des prêtres [curieusement, Ruge omet les prêtres dans sa citation, malgré sa propre critique de l'aliénation sous sa double forme religieuse et nationaliste ${ }^{15}$ ], des maitres et des serviteurs, des jeunes gens et des adultes certes; mais pas un homme [le texte allemand répète à quatre reprises "keine Menschen», "pas d'êtres humains»; ici comme souvent, la traduction de Jaccottet reste trop éloignée du texte et des réalités historiques et intellectuelles allemandes, en particulier si l'on considère l'importance de la notion d'être humaindans l'humanisme jeune-hégélien]. On croirait voir un champ de bataille couvert de bras, de mains, de membres pêle-mêle, où le sang de la vie se perd lentement dans les sables.» (267) ${ }^{16}$.

Voilà donc Hypérion et Hölderlin intégrés, cinq ans avant les révolutions de 1848 - Ruge siégera à l'extrême gauche du Parlement de Francfort -, à une critique philosophique et politique radicale de la misère allemande ${ }^{17}$, c'est-à-dire d'abord et surtout de l'incapacité de la nation allemande à mettre en œuvre, de façon autonome, sa propre libération politique par le biais d'une révolution du type 1789 redoublée, en France, en juillet 1830. Le roman de Hölderlin s'ouvre en effet, on l'a vu, sur une

13 Cité dans G. HERWEGH, "Ein Verschollener", in Herweghs Werke in drei Teilen, hrsg. von H. TARDEL, Berlin, Leipzig, Vienne et Stuttgart, Bong \& Cie, s. d., 2. Teil, Gedichte und kritische Aufsätze aus den Jahren 1839-1840, p. 78-81, p. 80.

14 Sur la discussion Ruge-Marx en 1843-1844, voir L. CALVIÉ, Aux origines du couple franco-allemand. Critique du nationalisme et révolution démocratique avant 1848. Arnold Ruge, Toulouse, PUM, 2004.

15 Voir ibid.

16 Cité dans A. RUGE, Sämtliche Werke, 2. Auflage, 9. Band, Polemische Briefe, Mannheim, J.P. Grohe, 1848, p. 114-115.

17 Voir Misère allemande/Deutsche Misere (sous la direction de L. CALVIÉ et F. GENTON), Chroniques allemandes 7 (1998-1999). 
critique de la philosophie idéaliste allemande - celle de Fichte en l'occurrence -, présentée comme incapable de donner une réponse adéquate au malheur humain étroitement uni à la misère politique, et s'achève, ou presque, sur une critique plus générale des Allemands comme peuple, ou comme nation, décrits comme incapables d'accéder à l'état d'humanité, et donc de liberté individuelle et politique.

Le caractère durement radical de la position de Hölderlin sur l'opposition entre les peuples naturellement libres - les anciens Athéniens et sans doute aussi les Français, dont la Révolution «rejoue» une Antiquité d'ailleurs plus romaine que grecque et paraît capable de recréer l'harmonie perdue de la vie antique - et les peuples naturellement inaptes à cette même liberté - les «Égyptiens» et les «Fils du Nord», parmi lesquels se rangent certainement les Allemands - trouve son corollaire dans la lettre sur l'inhumanité des Allemands qui clôt presque le roman et l'éclaire rétrospectivement. Le radicalisme de principe au sujet du rapport des différents peuples à la liberté n'est ainsi que l'autre face d'un pessimisme profond, existentiel et historique tout à la fois, touchant à l'impuissance des Allemands, comme individus et comme nation, à accéder à la liberté individuelle et politique, c'est-à-dire aussi au bonheur et à la beauté.

Cette constatation trouve peut-être une illustration complémentaire dans l'examen des réactions négatives de la plupart des spécialistes allemands ${ }^{18}$ à la vision très politique de Hölderlin proposée, on l'a vu, en 1968-1969 - la date n'est pas indifférente - par Pierre Bertaux, à la suite d'ailleurs du critique marxiste hongrois Georg Lukács, dès 1934-1935 et, en 1959, d'un germaniste français, Maurice Delorme ${ }^{19}$, né en 1926 et engagé dans les Forces Françaises de l'Intérieur (F.F.I.), en 1943, à dix-sept ans. Plus généralement, les opinions plutôt réticentes de ces mêmes spécialistes allemands sur la dimension politiquement radicale de l'œuvre de Hölderlin, et de Hypérion en particulier, paraissent corroborer, négativement, l'interprétation avancée ici.

Un exemple suffira pour illustrer cela, celui d'Hypérion comme roman historique et politique «à clé» dans lequel la situation grecque de 1770 illustre et permet de comprendre, dans un langage en quelque sorte codé, celle de la dernière décennie du

18 Voir G. MERLIO, “Les chassés-croisés franco-allemands dans la «réception" de l'œuvre de Hölderlin", in Bordeaux au temps de Hölderlin (sous la direction de G. MERLIO et N.P ELLETIER), Berne, P. Lang, 1997, p. 311-332; G. MIETH, "Ein Rückblick auf öffentliche Hölderlin-Ehrungen 1970", Texte zur Literatur, Heft 1 (Im Zwielicht des Jahrhunderts. Beiträge zur Hölderlin-Rezeption, hrsg. von A. KLEIN, G. MIETH und K.P EZOLD), Rosa-Luxemburg-Verein, Leipzig, s. d. [1994], p. 49-65.

19 Voir G. LUKÁCS, "Hölderlins Hyperion", Internationale Literatur 6 (1935), p. 96-110 (traduction française: Les Cabiers d'Action 2 [1946], p. 28-47 et in G. LUKÄCS, Goethe et son époque, trad. de l'allemand par L. GOLDMANN et M. BUTOR, Paris, Nagel, 1949) et M. DELORME, Hölderlin et la Révolution française, Monaco, Éditions du Rocher, 1959. 
XVIII siècle en Allemagne. Analysant la "constellation historique» du roman de Hölderlin, un spécialiste allemand, Gerhard Kurz, affirme, à propos du sens de la description du siège, de la prise et du pillage de Mystra en 1770 par les Grecs associés aux troupes russes, que, pour Hölderlin, les armées révolutionnaires françaises menaient davantage des guerres de conquête que de libération ${ }^{20}$. Certes, dans le roman, Hypérion dit son écœurement face aux violences et aux pillages auxquels il a assisté. Mais si les Grecs insurgés, qui sont en Grèce chez eux, «représentent», du moins si l'on suit Kurz, les armées françaises en Allemagne, quelles forces et quels peuples peuvent bien alors "représenter» les Turcs, puissance occupante, et les Russes, alors en guerre contre l'Empire ottoman et donc alliés au moins objectifs des insurgés grecs?

Beaucoup plus cohérente paraît ici l'interprétation proposée à la fin des années 1950 par Maurice Delorme. Celui-ci, en effet, affirmait :

«... on peut voir un symbole dans le fait que ces soit aux compatriotes d'Hypérion (où nous avons plus d'une fois reconnu les Allemands de la fin du XVIII siècle) que Hölderlin adresse les critiques visant les révolutionnaires français [...]. Hölderlin en avait à la passivité des Allemands qui, n'ayant pas su utiliser la présence des armées révolutionnaires pour faire leur révolution eux-mêmes, ont justifié partiellement l'attitude hostile des soudards français [...]»

Et il ajoutait, à propos de la décision d'Hypérion, face aux inconséquences et au désordre des insurgés grecs, de rejoindre la flotte russe:

«On entrevoit là un état d'esprit qui a dû être souvent celui de Hölderlin: se mettre au service des Français, purement et simplement, malgré les imperfections flagrantes de leur révolution.» 21

Dans l'interprétation avancée par Delorme, les Grecs de 1770 «représentent» les Allemands des années 1790, les troupes russes «représentent» les troupes françaises en Allemagne et les Turcs ou Ottomans, enfin, «représentent» l'Ancien régime allemand et européen, injuste et oppressif. Sans doute peut-on apercevoir dans la cohérence de cette interprétation - nouvel aller-retour entre le présent vécu et la référence au passé historique - le résultat de l'expérience politique précoce de Delorme, celle du tout jeune résistant à l'ordre nazi, dit «nouveau», instauré en Allemagne, en France et dans la plus grande partie de l'Europe de 1940 à 1944.

De manière analogue, dans le texte d'Hypérion rédigé et publié de 1792 à 1799, la double référence hölderlinienne au passé lointain et idéalisé de la Grèce antique et au passé récent de l'insurrection grecque de 1770 prenait appui sur une perception et une compréhension immédiates des affrontements de l'époque révolutionnaire. Et, en

20 G. KURZ, "Friedrich Hölderlins Roman «Hyperion» oder «der Eremit in Griechenland»", Bad Homburger Hölderlin-Vorträge 1986-1987, p. 26-35, p. 32.

21 DELORME, Hölderlin, p. 168-169. 
retour, cette perception et cette compréhension du phénomène révolutionnaire étaient marquées par la double référence à l'Antiquité grecque et, plus accessoirement sans doute, à la révolte de la Grèce moderne contre la domination ottomane en 1770.

Lucien CALVIÉ

Université de Toulouse-Le Mirail

"La Pléiade» $n^{\circ} 2$

506, route de Grézac

F-34700 Lodève 